\title{
ACCURATE ESTIMATION OF ORIENTATION PARAMETERS OF UAV IMAGES THROUGH IMAGE REGISTRATION WITH AERIAL OBLIQUE IMAGERY
}

\author{
F. A. Onyango*, F. Nex, M. S. Peter, P. Jende \\ Dept. of Earth Observation Science, Faculty ITC, University of Twente, The Netherlands - \\ f.arthur.onyango@gmail.com, \{f.nex, m.s.peter, p.1.h.jende\}@utwente.nl
}

KEY WORDS: Unmanned Aerial Vehicles, Aerial Oblique Imagery, Image Matching, Image Orientation

\begin{abstract}
:
Unmanned Aerial Vehicles (UAVs) have gained popularity in acquiring geotagged, low cost and high resolution images. However, the images acquired by UAV-borne cameras often have poor georeferencing information, because of the low quality on-board Global Navigation Satellite System (GNSS) receiver. In addition, lightweight UAVs have a limited payload capacity to host a high quality on-board Inertial Measurement Unit (IMU). Thus, orientation parameters of images acquired by UAV-borne cameras may not be very accurate.

Poorly georeferenced UAV images can be correctly oriented using accurately oriented airborne images capturing a similar scene by finding correspondences between the images. This is not a trivial task considering the image pairs have huge variations in scale, perspective and illumination conditions. This paper presents a procedure to successfully register UAV and aerial oblique imagery. The proposed procedure implements the use of the AKAZE interest operator for feature extraction in both images. Brute force is implemented to find putative correspondences and later on Lowe's ratio test (Lowe, 2004) is used to discard a significant number of wrong matches. In order to filter out the remaining mismatches, the putative correspondences are used in the computation of multiple homographies, which aid in the reduction of outliers significantly. In order to increase the number and improve the quality of correspondences, the impact of pre-processing the images using the Wallis filter (Wallis, 1974) is investigated. This paper presents the test results of different scenarios and the respective accuracies compared to a manual registration of the finally computed fundamental and essential matrices that encode the orientation parameters of the UAV images with respect to the aerial images.
\end{abstract}

\section{INTRODUCTION}

During the last decades, Unmanned Aerial Vehicles (UAVs) have been used to acquire high resolution imagery for geoinformation applications. They provide a low-cost alternative to the traditional airplanes as platforms for spatial data acquisition (Nex \& Remondino, 2014). They also tend to have high repeatability and flexibility in data acquisition making them popular platforms for image acquisition. Additionally, UAVs acquire images that have a Ground Sampling Distance (GSD) of up to $1 \mathrm{~cm}$, which is relatively high compared to images taken by manned aircrafts.

However, UAV images often have poor georeferencing information due to the low quality on-board Global Navigation Satellite System (GNSS). To add to that, most UAVs have a limited payload capacity to host a high quality on-board Inertial Measurement Unit (IMU) to ascertain accurate orientation parameters.

A crucial part in trying to solve this problem involves image registration to identify a sufficient number of tie points, which are used to retrieve the orientation parameters of the UAV images with respect to the aerial images whose orientation parameters are known. There exist different algorithms for image registration, because the images being registered may have different characteristics in terms of resolution, geometry and radiometry (Shan et al. 2015). Different surveys (Brown, 1992; Zitová \& Flusser, 2003) have been done on image registration and these surveys show that the different characteristics of images ought to be accounted for when developing a suitable registration algorithm.
The registration procedure presented in this paper relies on stateof-the-art algorithms for feature extraction of distinct keypoints between an image pair. The algorithms ought to be invariant to differences in scale, rotation, illumination and viewpoints. Such algorithms are, e.g. KAZE (Alcantarilla et al. 2012), AKAZE (Alcantarilla, Nuevo, \& Bartoli, 2013), LATCH (Levi \& Hassner, 2015) and ORB (Rublee \& Bradski, 2011).

This paper aims at addressing the problem of automatically registering oblique UAV images to airborne oblique images by presenting a matching strategy that relies on the use of the AKAZE interest operator, which is used due to its invariance to large scale differences between our images of interest. Default settings used in AKAZE do not yield suitable keypoints for successful matching, hence the need to adjust the parameters, such as the number of octaves and the detection threshold.

Since the number of detected features decays from lower to higher octaves, the detection threshold is lowered to have sufficient features that have a good distribution in the matching octaves of the images.

The data set used in this paper is a sample of images availed to researchers in the framework of the multi-platform photogrammetry benchmark (Nex et al. 2015) undertaken by a scientific initiative of ISPRS and EuroSDR. Figure 1 shows a sample pair used in our experiments. The great variations in scale between the pair is evident, making the registration process a challenge.

The following section gives a brief literature review on similar research work already done. This is followed by section 3 , which outlines the methodology adopted in our research work.

\footnotetext{
${ }^{*}$ Corresponding author
} 

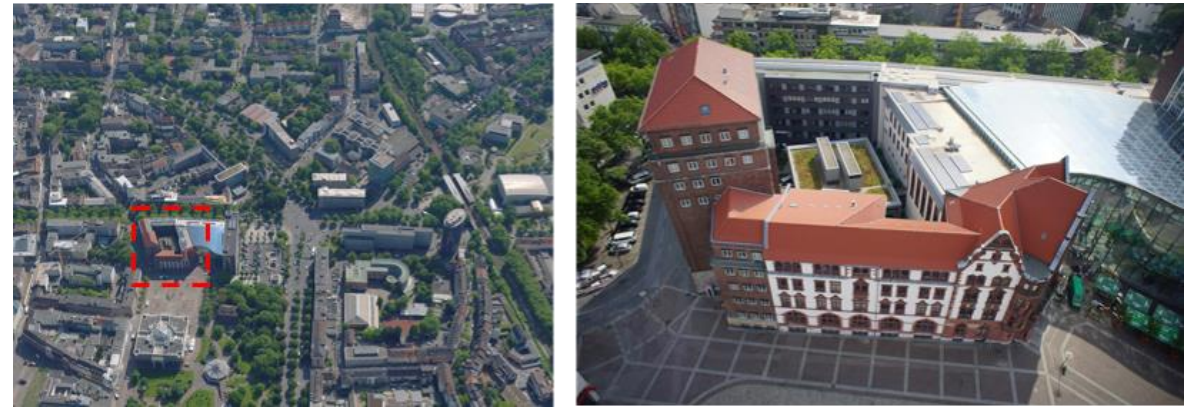

Figure 1. A pair of test images captured in Dortmund, Germany. Left: Aerial oblique. Right: UAV oblique image

The subsequent section presents the experimental results and finally the paper is concluded in section 5 where future research work is outlined.

\section{RELATED WORKS}

Various authors have presented different strategies to register images with different resolutions. For instance, Geniviva et al. (2014) proposed an automated registration technique that could be used to improve the positional accuracy of oblique UAV images using orthorectified imagery. The technique implemented the A-SIFT (Morel \& Yu, 2009) algorithm to find correspondences between the image pairs. A-SIFT was used due to its ability to vary the camera-axis parameters in order to simulate all possible views. However, this technique makes the algorithm computationally expensive.

More recently, Koch et al. (2016) proposed a new method to register nadir UAV images and nadir aerial images. An investigation was done to assess the viability of using SIFT (Lowe, 2004) and A-SIFT. The authors concluded that these methods did not yield satisfactory results because the images to be matched had a large difference in scale, rotation and temporal changes of the scene. This led to the proposed method, which used a novel feature point detector, SIFT descriptors, a one-tomany matching strategy and a geometric verification of the likely matches using pixel-distance histograms.

Jende et al. (2016) proposed a novel approach for the registration of Mobile Mapping (MM) images with high-resolution aerial nadir images. The approach involves using a modified version of the Förstner operator to detect feature keypoints only in the aerial ortho-image. The feature keypoints are then back-projected into the MM images. A template matching strategy is used to find correspondences as opposed to using feature descriptors. The approach was compared to combinations of AGAST detector \& SURF descriptor as well as Förstner detector \& SURF descriptor.

Gerke et al. (2016) performed experiments to investigate on how current state-of-the-art image matching algorithms perform on similar platform and across platform images such as UAV images and terrestrial images and concluded that standard image matching algorithms suffer from differences in illumination and geometry between image pairs.

Previous research methods do not offer reliable strategies for image registration between UAV images and aerial oblique images. This research proposes a reliable method to accurately register UAV images to already georeferenced aerial oblique images.

\section{METHOD}

Figure 2 depicts a flowchart showing an overview of the strategy adopted to develop our proposed image registration algorithm.

The following sub sections give detailed explanations to the key components of the proposed methodology.

3.1 Feature extraction: In order to restrict the search area for potential matching features in the aerial image, the availed orientation parameters of the input images were exploited to create a search window within the area of overlap in the aerial image. This was done by back-projecting the UAV image location into the aerial image and later on creating a 1000 by 1000 pixel search window around the back-projected point.

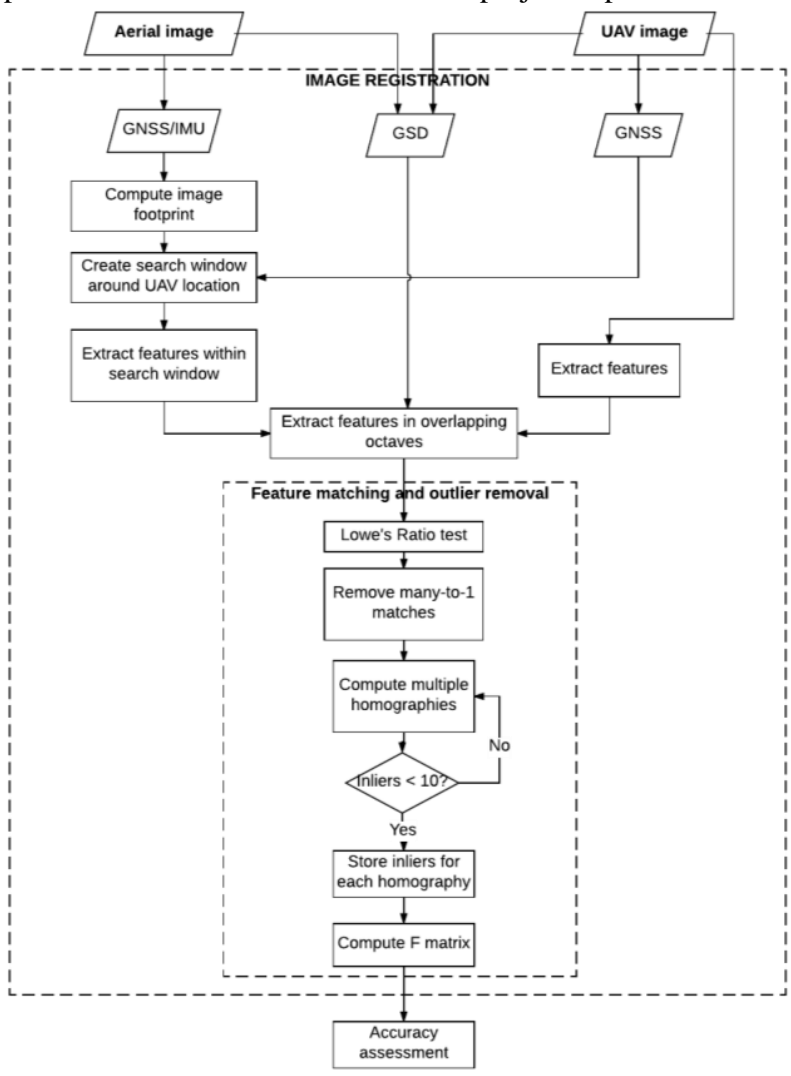

Figure 2. Flowchart showing an overview of the proposed method

AKAZE features are extracted within the search window of the aerial image and the entire UAV image. We use the AKAZE interest operator due to its invariance to scale and ability to preserve boundary features, thereby detecting more distinct 
features for matching. Moreover, SIFT, SURF (Bay et al. 2008), KAZE, BRISK (Leutenegger, Chli, \& Siegwart, 2011), and BRIEF (Calonder et al. 2010) were tested and AKAZE gave better results.

The number of octaves in the aerial image is then adjusted to two while the default number of four octaves is retained in the UAV image. The GSD ratio between the image pair is used to extract only features from the third and fourth octaves of the UAV image while features from the first and second octaves are discarded, because they are unlikely to match due to huge differences in scale.

Since the number of detected features decays from lower octaves to higher octaves and the higher octaves of the UAV image yield potential features for successful matching, the Hessian threshold which determines which features are to be detected is lowered progressively from the default value to detect a sufficient number of features in the higher octaves of the UAV image.

3.2 Feature matching: Brute force hamming distance (Hamming, 1950) is used to match the corresponding features due to the binary nature of the AKAZE descriptor. The putative matches contain a set of wrong matches that need to be discarded. Lowe's ratio test (Lowe, 2004) is employed to select the best matches and significantly reduce the number of outliers. The ratio test suggests that two closest matches are unlikely to share the same descriptor distance. If the descriptor distance ratio is above a certain threshold, then the matches are removed. If the distance ratio is below the predefined threshold, then the match with the lower descriptor distance is retained.

3.3 Multiple homographies: Feature matching alone is not able to provide a set of reliable correspondences, and many mismatches are still visible in the images. The direct estimation of a fundamental matrix using RANSAC for outlier removal does not yield reliable results. It was therefore decided to employ the computation of multiple homographies to filter out wrong matches in every iteration. Zuliani, Kenney, \& Manjunath, (2005) used a similar approach and they called it multiransac.

The computation of a homography between a pair of images is dependent on planar elements in a scene, and it is therefore suitable for images capturing urban environments. The buildings have structured surfaces with varying shapes and orientations making them multi-planar. Figure 3 illustrates this concept.

With this hypothesis in mind, multiple homographies are computed iteratively using the putative matches, earlier computed, as the only input. To derive a homography, at least four point pairs are required. RANSAC is used to look for points conforming to a homography. The first iteration takes the whole set of putative matches and computes the first homography. The inliers are stored while the outliers are used in the next iteration to compute the second homography. This is done iteratively until no more inliers are found. A condition is set to stop the iteration, whenever less than ten points are detected to compute a homography. This choice was made to avoid the computation of degenerate sets of inliers.

3.4 Fundamental (F) and Essential (E) matrices: The next step involved the computation of a global geometric relationship that exists between the image pairs. This is done by computing an $\mathrm{F}$ matrix using the eight point algorithm (Longuet-Higgins, 1981) that implements the use of RANSAC to remove any possible outliers that may have been computed as inliers during the computation of multiple homographies.

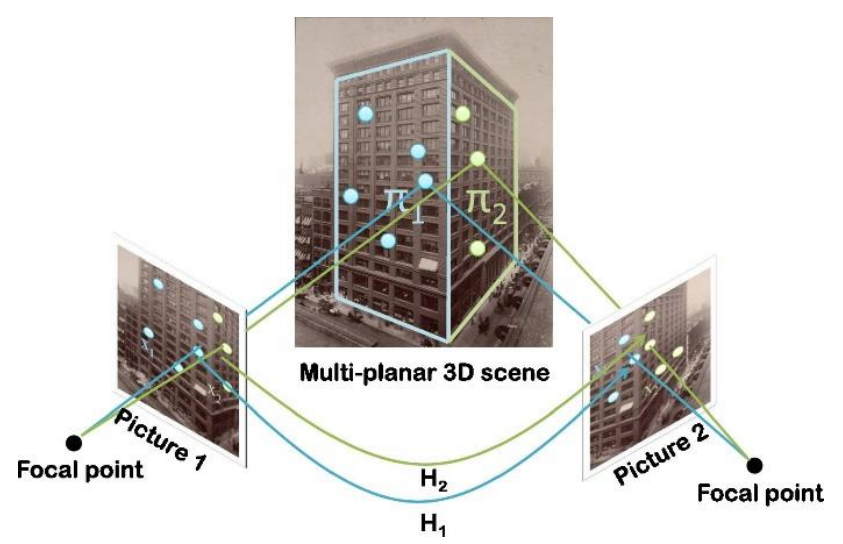

Figure 3. A building scene represented as having two planes. Homologous points from each plane have a homography mapping (Szpak et al. 2014)

The computation of the F matrix becomes suitable whenever the cameras' interior orientation parameters are unknown. On the other hand, since the orientation parameters of the oblique camera are precisely known and the UAV camera parameters can be approximated to a certain extent, the problem can be reduced to an E-matrix estimation only. This entails reducing the number of parameters to estimate and potentially leads to a better result.

3.5 Accuracy evaluation: Accuracy evaluation is done by making use of the epipolar constraint. Corresponding epipolar lines are computed and metric distances from these lines to their corresponding points are derived. An ideal case will result to distances equalling to zero, but in reality, this might not always be the case possibly due to localisation errors encountered during feature detection. Computation of the average residual error, as shown in equation 1 , is used to assess the accuracy of the F matrix in mapping point features from the aerial image to corresponding epipolar lines on the UAV image. The average residual error is compared with the average residual error computed from manually identified matches throughout the scene.

$$
\frac{1}{N} \sum_{i}^{N} d^{\prime}\left(x_{i}^{\prime}, F \hat{x}_{i}\right)
$$

Where $N$ is the total number of matching points and $d(x, F x)$ is the distance between a point to its corresponding epipolar line in the other image.

Since the objective is to register the UAV image to the aerial image, the residual errors between matched points are computed in the UAV image.

\section{EXPERIMENTS}

In order to test the developed registration algorithm, we use eight sample pairs of the benchmark images acquired in the city centre of Dortmund, Germany. The image pairs were selected, because they comprise of buildings posing a challenge in registration by having repetitive patterns. In addition, the image pairs reflect great differences in viewpoint, illumination and scale.

The eight pairs of images have different combinations and capture different scenes in order to test the reliability of the developed algorithm under different scenarios. 
All experiments were conducted in MATLAB using integrated OpenCV functions together with MATLAB built-in functions.

\subsection{Experimental results}

This section gives an illustration of the results derived after testing the developed algorithm on different image pairs. A good number of images were successfully matched, whereas a few image pairs did not return satisfactory results.

\section{Pair 1:}

Figure 4 shows an image pair, both taken at a viewing angle of 45 degrees. 206 correct matches were computed without a single outlier. This is because the ratio test provided a robust solution in retaining distinct matches. Moreover, computing multiple homographies proved reliable in filtering out the few mismatches that evaded the ratio test, because multiple planes present in the scene were estimated successfully.

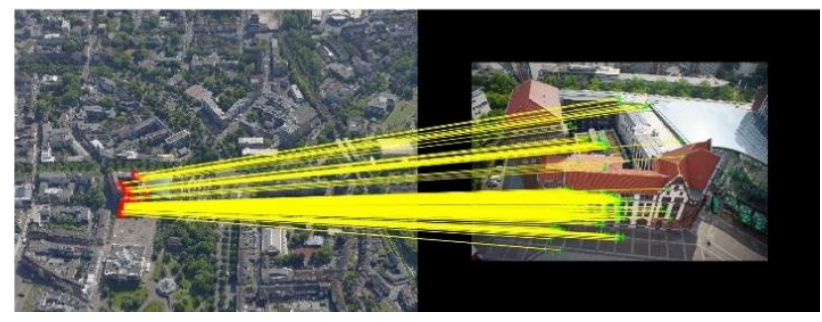

Figure 4. 206 correct matches of the Stadthaus building scene

\section{Pair 2:}

Figure 5 shows the results achieved when a UAV image, captured at an almost horizontal angle to the building, was matched with an aerial oblique image with a vertical viewing angle of 45 degrees. 41 correct matches were computed and they were only on the façade. The facade has a good texture, a phenomenon that presumably led to a successful registration.

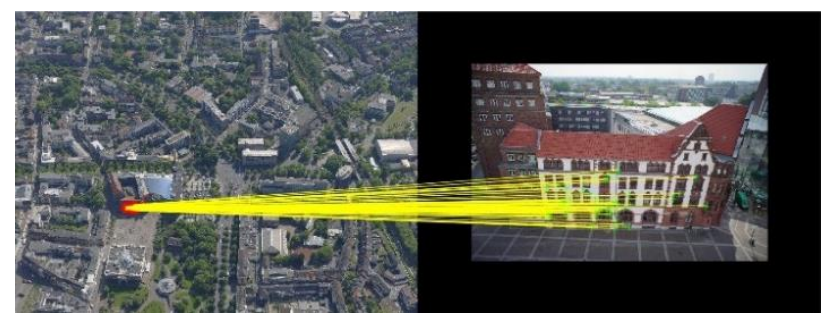

Figure 5. 41 correct matches of the Stadthaus building scene

\section{Pair 3:}

The result depicted in Figure 6 shows 76 correct correspondences. The configuration is almost similar to the one in pair 1, but the only difference is that the UAV image had a slightly different vertical viewing angle of about 10 degrees and captured a portion of the side of the building. Despite this difference in viewpoint, the registration was still successful.

\section{Pair 4:}

Pair 4 shows a challenging configuration where there was a huge difference of approximately 45 degrees in the horizontal viewing angles between the UAV camera and the aerial camera. There was also a difference in the vertical viewing angles between the two cameras with the aerial camera having a viewing angle of 45 degrees and the UAV camera having a viewing angle of about 10

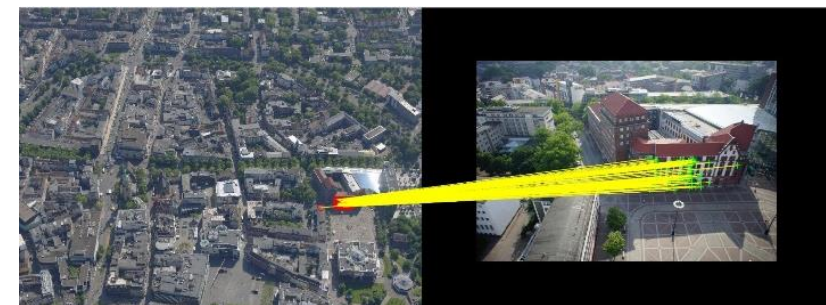

Figure 6.76 correct matches of the Stadthaus building scene

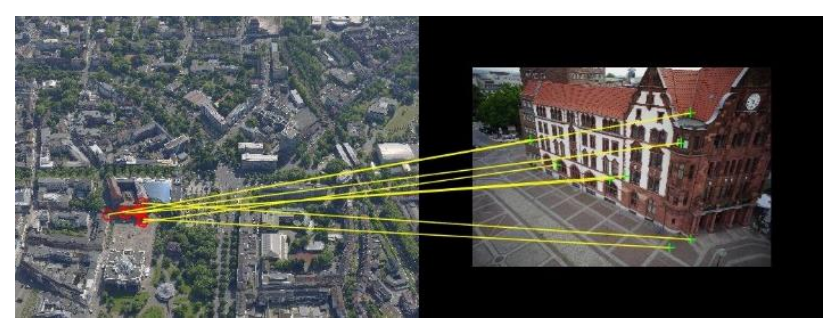

Figure 7. No correct match due to drastic differences in viewing angles of the Stadthaus building scene

degrees. Unfortunately, no correct matches were computed as shown in Figure 7. This can be attributed to the extreme differences in viewing angles between the two images that causes some features to be distorted and occluded in the UAV image, thus hampering the process of detecting corresponding features in both images.

\section{Pair 5:}

Figure 8 shows the registration results of a pair of images comprising of a different building scene that has many repetitive features. Both cameras captured the respective images at a viewing angle of 45 degrees. 229 correct matches were computed throughout the scene. The scene captured has a favourable texture that is suitable for matching. In addition, multiple planes were present in the scene, which contributed to a successful result.

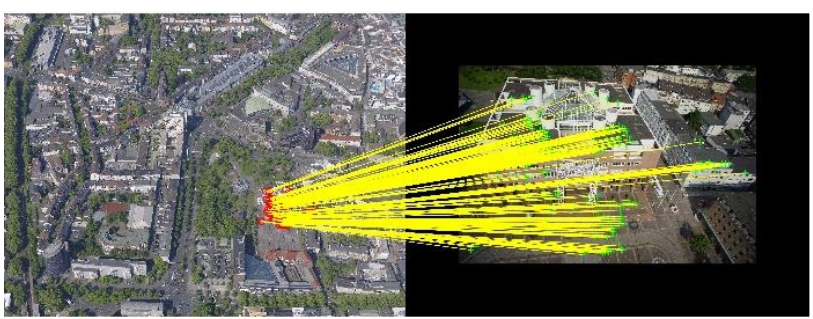

Figure 8. 229 correct matches of the Rathaus building scene

\section{Pair 6:}

Pair 6 represents a slightly similar scenario to pair 5 with the only difference being the viewing angle of the UAV camera, which is about 5 degrees off nadir. Figure 9 shows a successful registration of 155 correct matches, all of which are on the rooftop of the Rathaus building, since this part was mostly captured by the UAV camera, which was looking almost vertically downwards. Despite having repetitive features, the images were successfully matched. 


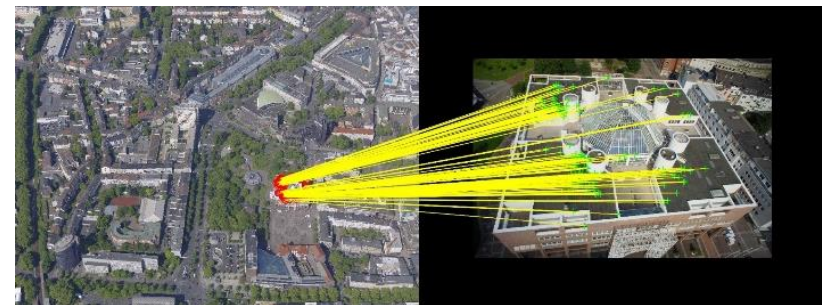

Figure 9. 155 correct matches of the Rathaus building scene

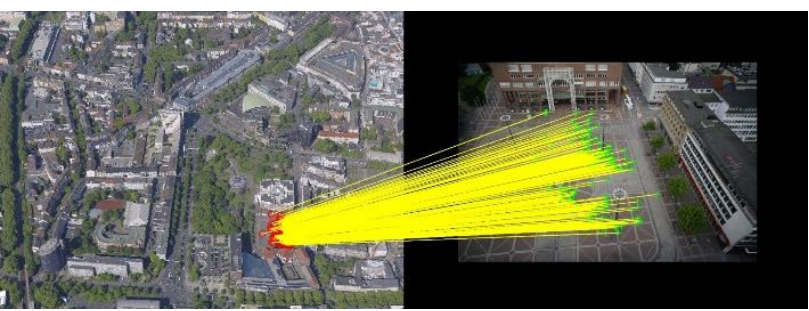

Figure 10. 234 correct matches of the Friedensplatz scene

\section{Pair 7:}

The UAV image used in pair 7 comprises mainly the open space in front of the Stadthaus and the Rathaus buildings. Figure 10 illustrates 234 correct matches. The successful registration can be attributed to the good texture and the pattern of the ground area. In addition, the similar viewing angles between the cameras, i.e. 45 degrees, also contributed to the correct correspondences.

\section{Pair 8:}

The last pair shows a configuration where the registration failed. This configuration has a drastic difference in viewing angles between the two cameras, i.e. both cameras were facing different sides of the Rathaus building - the aerial camera faced the south façade and the UAV camera faced the east façade. Figure 11 depicts the mismatches computed.

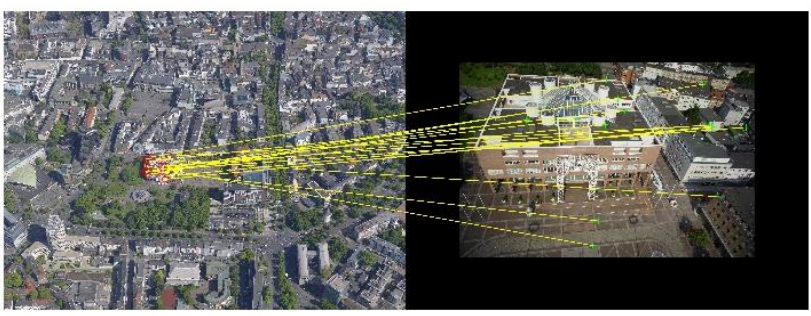

Figure 11. Mismatches between different views of the Rathaus building

\subsection{Accuracy evaluation}

Table 1 shows an analysis of the residual errors of the respective F matrices computed, both automatically and manually, for the eight image pairs. In addition to the average residual errors, the minimum and the maximum residual errors are also given.

For the image pairs that were successfully registered, the average residuals computed are all below five pixels on the UAV image. The same applies to the average residual errors computed from manual registration. These values are slightly lower than the values computed automatically with an exception in pair 4 , which gave an average residual error of 65.86 pixels from automatic registration. No residual errors were computed for pair 8 , because the image pair barely had corresponding features that were easily distinguishable.

\begin{tabular}{|c|r|r|r|r|r|r|}
\hline \multirow{2}{*}{ Pair } & \multicolumn{6}{|c|}{ Residual error (pixels) } \\
\cline { 2 - 8 } & \multicolumn{1}{|c|}{ Automatic registration } & \multicolumn{2}{c|}{ Manual registration } \\
\cline { 2 - 7 } & \multicolumn{1}{|c|}{ Mean } & \multicolumn{1}{c|}{ Min } & \multicolumn{1}{c|}{ Max } & \multicolumn{1}{c|}{ Mean } & \multicolumn{1}{c|}{ Min } & \multicolumn{1}{c|}{ Max } \\
\hline pair 1 & 4.91 & 0.11 & 27.46 & 3.12 & 0.04 & 15.35 \\
\hline pair 2 & 3.45 & 0.13 & 10.18 & 3.26 & 0.44 & 10.29 \\
\hline pair 3 & 2.53 & 0.02 & 10.91 & 2.42 & 0.03 & 9.92 \\
\hline pair 4 & 65.86 & 0.17 & 449.41 & 7.75 & 0.45 & 29.9 \\
\hline pair 5 & 4.43 & 0.01 & 39.22 & 3.67 & 0.05 & 11.09 \\
\hline pair 6 & 4.61 & 0.01 & 28.83 & 3.35 & 0.3 & 19.25 \\
\hline pair 7 & 2.98 & 0.02 & 27.03 & 2.04 & 0.21 & 12.23 \\
\hline pair 8 & 24.85 & 0.35 & 84.85 & - & - & - \\
\hline
\end{tabular}

Table 1. Summary of the residual errors of the respective F matrices computed automatically and manually

\subsection{Influence of Wallis filter}

An additional experiment was conducted to assess the impact of pre-processing the images using the Wallis filter. We chose pair 1 due to the variation in illumination between the images. Figure 12 shows the image pair with a zoomed in version of the aerial image around the area of overlap. It is evident that the aerial image has less contrast compared to the UAV image. This is most likely due to the different daytimes or days that the images were acquired.

The Wallis filter (Wallis, 1974) has five adjustable parameters (size of the filter, target mean, target standard deviation, contrast and brightness factors). The main objective is to apply the filter on both images to reduce the differences in illumination. Since the images also have different scales, it was decided to readjust the size of the filter on the UAV image while the filter size on the aerial image was kept at a constant (default value). This choice was made because the image pair have varying resolutions hence the need to use varying filter sizes for both images.

Although the overall result did not improve significantly, it was observed that upon increasing the filter size, the number of matches increased progressively as shown in Figure 13. Figure 14 shows the result of matching image pair 1 after applying the Wallis filter with a filter size of 40 by 40 pixels on the aerial image and a filter size of 200 by 200 pixels in the UAV image.
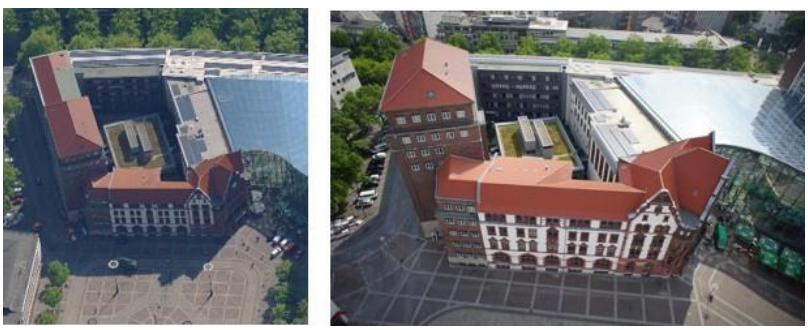

Figure 12. Pair 1. Left: Zoomed in aerial image. Right: UAV image 


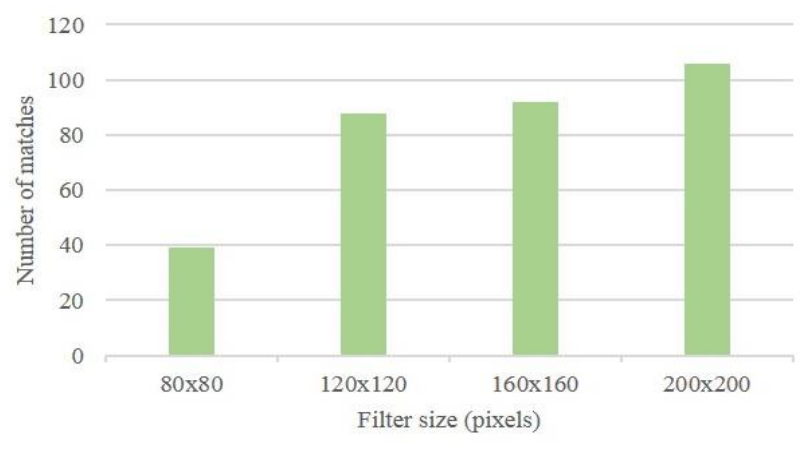

Figure 13. Increasing number of matches as the Wallis filter size is increased on the UAV image

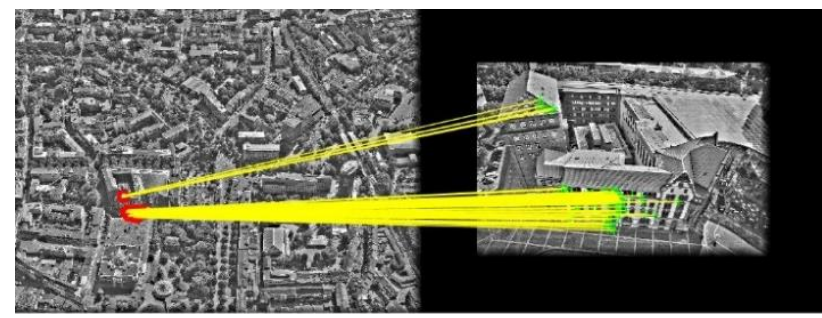

Figure 14. 106 matches after applying Wallis filter on the image pair

\section{DISCUSSION}

The developed methodology has showed reliable results in most of the performed tests. Scale changes can be efficiently handled by modifying the number of octaves considered in the extraction of salient features while multiple homographies can cope with the larger percentage of outliers that usually occur with multi resolution images. The repetitive nature of some buildings does not prevent the correct detection of several corresponding features within the image pairs.

However, the achieved results show that the registration procedure can reliably work when the differences in looking directions are less than 25 degrees. The number of matches is usually related to the differences in viewing directions of the images. As it could be expected, the presence of well-textured surfaces can greatly influence the number of corresponding features and their distribution throughout the image.

The performed tests have then showed that the residuals of the automated procedure are of the same order to those of the manual registration. The residuals are 3-5 cm GSD (of the UAV images) and this corresponds to $1 \mathrm{~cm}$ GSD of the aerial image, which is a fairly satisfying result. The only exceptions are when the registration is not successful. In these cases, high values are a clear indication of the failure of the procedure.

The Wallis filter does reduce the illumination variations but it does not increase the number of matched features. However, increasing the filter size for the UAV image has the consequence to increase the number of matched features. This might be attributed to the different resolutions of the images and that reflects the need to use different filter sizes to compensate for their different resolutions.

\section{CONCLUSIONS AND FUTURE OUTLOOK}

The main aim of this paper was to present a strategy to accurately orient UAV images via feature matching with already georeferenced aerial oblique images. The image pairs adopted in the tests presented challenging scenarios due to differences in scale, geometry and even lighting conditions.

The achieved results looked promising in most of the cases, showing the suitability of the state-of-the-art interest operators for this task. Nonetheless, the developed procedure is still exploiting the availed orientation parameters of the image pairs as input to restrict a search area for candidate features in the area of overlap in the aerial image.

Many tests have been previously performed using different interest operators (Onyango, 2017) and AKAZE showed to outperform all the others in most of the cases. Some strategies were then adopted on the original AKAZE implementation to be more effective in registering images with different resolutions. A first successful strategy was to lower the Hessian threshold in order to yield a sufficient number of features in the higher octaves of the UAV images. The respective GSDs of the images were used to automatically infer which octaves in both the images had a similar resolution and which features detected in the octaves of the UAV image looked more similar to the features in the octaves of the aerial image. The implementation of Lowe's ratio test played an instrumental role in reducing the number of outliers in the matching step. Moreover, the computation of multiple homographies further reduced the number of outliers thereby necessitating the computation of a reliable $F$ matrix whose residual errors depicted acceptable accuracies apart from image scenarios that had extreme differences in geometry.

The limitations in matching features on images from very different viewing directions are mainly due to the current limits faced by interest operators. The differences in scale do not play a major role in this context.

For image pairs that did not achieve a good distribution of matches, other matching and image processing techniques can be exploited in an attempt to solve this problem. Such techniques include graph matching and image segmentation. Another promising solution could be represented by deep learning approaches as reported in (Altwaijry et al. 2016).

Next research would be to register between terrestrial images and already oriented UAV images. Here the scale difference between the image pairs are of a lower magnitude compared to the scale differences between aerial and UAV images. A challenge that might hamper the registration process is the differences in viewing angles that lead to geometrical differences between the images.

\section{ACKNOWLEDGEMENTS}

We thank ISPRS (Scientific Initiative) and EuroSDR for providing the data set under the framework of the multi-platform photogrammetry benchmark.

\section{REFERENCES}

Alcantarilla, P. F., Bartoli, A., \& Davison, A. J. (2012). KAZE features. Lecture Notes in Computer Science, 7577 LNCS(PART 6), 214-227. 
diffusion for accelerated features in nonlinear scale spaces. British Machine Vision Conference, 13.1-13.11.

Altwaijry, H., Trulls, E., Hays, J., Fua, P., \& Belongie, S. (2016). Learning to match aerial images with deep attentive architectures. Computer Vision and Pattern Recognition.

Bay, H., Ess, A., Tuytelaars, T., \& Van Gool, L. (2008). Speededup robust features (SURF). Computer Vision and Image Understanding, 110(3), 346-359.

Brown, L. G. (1992). A survey of image registration techniques. ACM Computing Surveys, 24(4), 325-376.

Calonder, M., Lepetit, V., Strecha, C., \& Fua, P. (2010). BRIEF : Binary robust independent elementary features. European Conference on Computer Vision (ECCV), 778-792.

Geniviva, A., Faulring, J., \& Salvaggio, C. (2014). Automatic georeferencing of imagery from high-resolution, low-altitude, low-cost aerial platforms. Geospatial InfoFusion and Video Analytics IV; and Motion Imagery for ISR and Situational Awareness II, 9089.

Gerke, M., Nex, F., \& Jende, P. (2016). Co-registration of terrestrial and UAV-based images - Experimental results, XL(February), 10-18. http://doi.org/10.5194/isprsarchives-XL3-W4-11-2016

Hamming, R. W. (1950). Error Detecting and Error Correcting Codes. Bell System Technical Journal, 29(2), 147-160.

Jende, P., Peter, M., Gerke, M., \& Vosselman, G. (2016). Advanced tie feature matching for the registration of mobile mapping imaging data and aerial imagery. The International Archives of the Photogrammetry, Remote Sensing and Spatial Information Sciences, XLI(July), 617-623.

Koch, T., Zhuo, X., Reinartz, P., \& Fraundorfer, F. (2016). A new paradigm for matching UAV- and aerial images. ISPRS Annals of Photogrammetry, Remote Sensing and Spatial Information Sciences, III(July), 12-19.

Leutenegger, S., Chli, M., \& Siegwart, R. Y. (2011). BRISK: Binary robust invariant scalable keypoints. Proceedings of the IEEE International Conference on Computer Vision, 2548-2555.

Levi, G., \& Hassner, T. (2015). LATCH: Learned arrangements of three patch codes, arXiv 1501.

Longuet-Higgins, H. C. (1981). A computer algorithm for reconstructing a scene from two projections. Nature, 293, 133135 .

Lowe, D. G. (2004). Distinctive image features from scale invariant keypoints. International Journal of Computer Vision, $60,91-110$.

Morel, J.-M., \& Yu, G. (2009). ASIFT: A new framework for fully affine invariant image comparison. SIAM Journal on Imaging Sciences, 2(2), 438-469.

Nex, F., Gerke, M., Remondino, F., Przybilla, H.-J., Bäumker, M., \& Zurhorst, A. (2015). ISPRS benchmark for multi-platform photogrammetry. ISPRS Annals of Photogrammetry, Remote Sensing and Spatial Information Sciences, II-3/W4(March), 135142 .

Nex, F., \& Remondino, F. (2014). UAV for 3d mapping applications: A review. Applied Geomatics, 6(1), 1-15.

Onyango, F. A. (2017). Multiresolution automated image registration (Master's thesis). Univerisity of Twente.

Rublee, E., \& Bradski, G. (2011). ORB: An efficient alternative to SIFT or SURF. Proceedings of the IEEE International Conference on Computer Vision, 2564-2571.

Szpak, Z. L., Chojnacki, W., Eriksson, A., \& Van Den Hengel, A. (2014). Sampson distance based joint estimation of multiple homographies with uncalibrated cameras. Computer Vision and Image Understanding, 125, 200-213.

Wallis, K. F. (1974). Seasonal adjustment and relations between variables. Journal of the American Statistical Association, 69(345), 18-31.

Zitová, B., \& Flusser, J. (2003). Image registration methods: A survey. Image and Vision Computing, 21(11), 977-1000.

Zuliani, M., Kenney, C. S., \& Manjunath, B. S. (2005). The multiransac algorithm and its application to detect planar homographies. Proceedings - International Conference on Image Processing, ICIP, 3, 153-156. 